\title{
"Exfoliative cytology in the diagnosis of cancer of the bronchus
}

\author{
S. W. A. KUPER \\ From the Brompton Hospital, London
}

It is proposed to divide this talk into three sectionssputum, blood, and pleural fluid. The first part outlines the usefulness of cancer diagnosis by sputum examination in routine pathological practice. The section on blood describes a research technique and attempts an assessment of the clinical significance of exfoliated tumour cells in human blood. Finally, the problem of identification of cancer cells in pleural fluid is discussed and a few as yet unproved methods are suggested for differentiating between mesothelial and tumour cells.

Much of the work reported here was carried out in cooperation with Dr. N. K. Wallace, of the Institute of Cancer Research, and Mr. R. A. Sellwood, of the Postgraduate Medical School of London, and it is hoped to publish the results fully in due course.

\section{SPUTUM}

During the winter of $1960-61$, Dr. K. F. W. Hinson and I, working at the Brompton Hospital, attempted to investigate some aspects of sputum examination as an aid to the diagnosis of carcinoma of the bronchus (Hinson, and Kuper, 1963). All the samples examined were from hospital patients, so that chest disease was nearly always present and cancer of the lung was often suspected. We made no attempt to prove that any individual patient had cancer, but simply attempted an unbiased assessment of all routine samples submitted. Clinicians were asked to ensure that an early morning sample of sputum was submitted but this was not always done and a certain proportion of the specimens contained food or consisted mainly of saliva. The specimens were usually examined on the same day, but if for any reason this was not feasible, they were placed in the refrigerator overnight. No preservatives or fixatives were used.

Each sputum sample was placed in a Petri dish on a black background and suitable fragments were picked out.

${ }^{1}$ A paper read at the first meeting of the British Society for Clinical Cytologists, 1962.
When present, white threads or bloodstained streaks were chosen. The samples selected were emulsified on slides with $1 \%$ methylene blue, and covered with large coverslips which were gently pressed until the sputum layer was of suitable thickness. No fixative was used, so that cells were not shrunken and their morphology was well preserved. If the pathologist was in doubt, the material was still fresh and further preparations could be made.

This technique has one serious disadvantage, however. Preparations are not permanent, and photographs were used to record all positive results.

No attempts were made in this survey to compare the advantages of examining fixed and unfixed material. It seems to us that no particular staining technique has any special merit and the one of choice is that with which one is fully familiar. Methylene blue has the virtue of speed, for preparations can be made in a few minutes.

At the time of this survey we had experimented with some liquefaction techniques, but in our hands they were of uncertain reliability. We were not satisfied that acridine orange fluorescence was of assistance in scrutinizing preparations. All the sputum samples were produced without the aid of deliberate respiratory irritants which have been found a useful adjunct by some workers.

All samples were presented simply with a serial number, in an effort to minimize bias by the observers. Each of us prepared two slides and scrutinized each slide for up to five minutes before discarding it as 'negative'. As soon as a slide was recorded 'positive', the search was discontinued. Most samples were recorded as 'positive' or 'negative' and we only rarely hid behind the heading 'doubtful'. When tumour cells were found, an attempt was made to identify the cell type. We never compared results until our two individual decisions had been separately recorded.

Figures 1 and 2 show characteristic examples of the types of cancer cells which are seen in the sputum of patients with carcinoma of the bronchus.

RESULTS Over 1,600 sputum samples were examined from 541 patients, of whom 229 had proved cancer of 


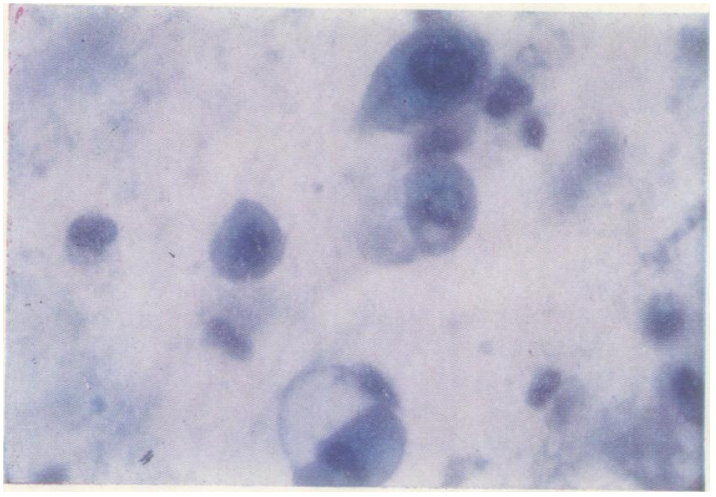

FIG. 1a. Squamous carcinoma cells in sputum

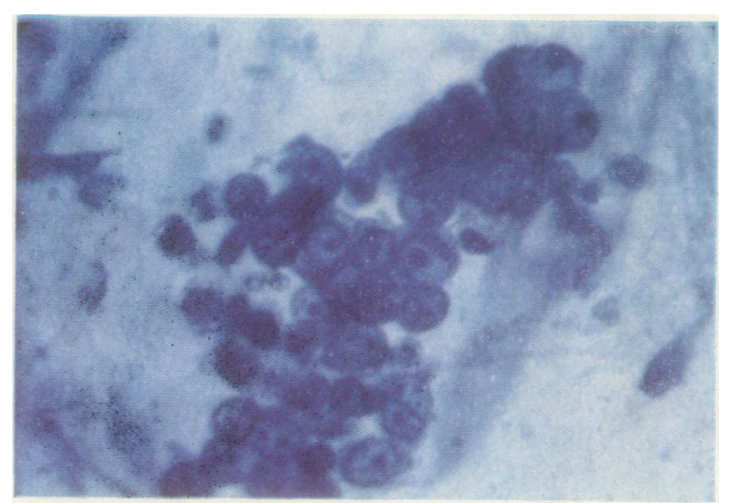

FIG. 2a. Undifferentiated carcinoma cells in sput:Im

the bronchus, or in whom the clinical diagnosis was confidently established. A further 10 patients had secondary cancer of the lung. By contrast with several other workers, we failed to detect tumour cells in this class of patient. In assessing the value of the results given, it is of some importance to remember that they refer only to the set of conditions outlined above and should not therefore be compared with other surveys in which the modes of assessment were different.

Of the 229 patients with carcinoma of the bronchus, one or both of the observers found tumour cells in $60 \%$. In the 70 patients with squamous carcinoma, the percentage positive rose to 67 ; with oat-cell carcinoma (14 patients) it fell to 36 . In the 77 patients in whom there was no histological assessment of cell type, the positive identification was made in $71 \%$. It is possible that this high figure merely reflects advanced cancer in patients in whom bronchoscopy or major surgery was considered impracticable and in whom necropsy was not per-

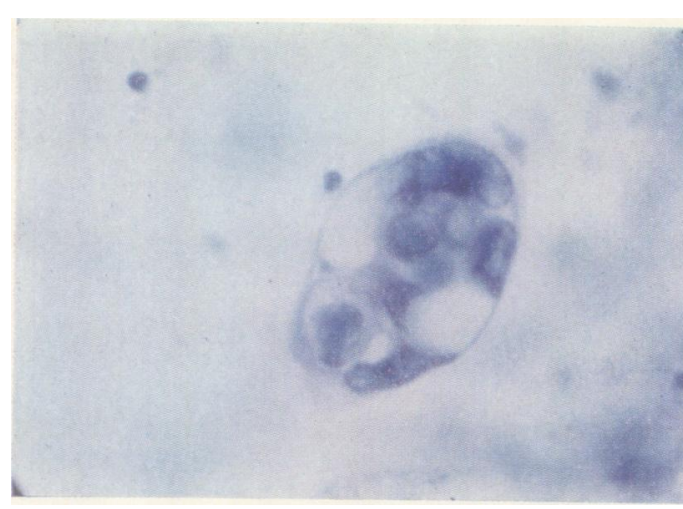

FIG. 1b. Adenocarcinoma in sputum

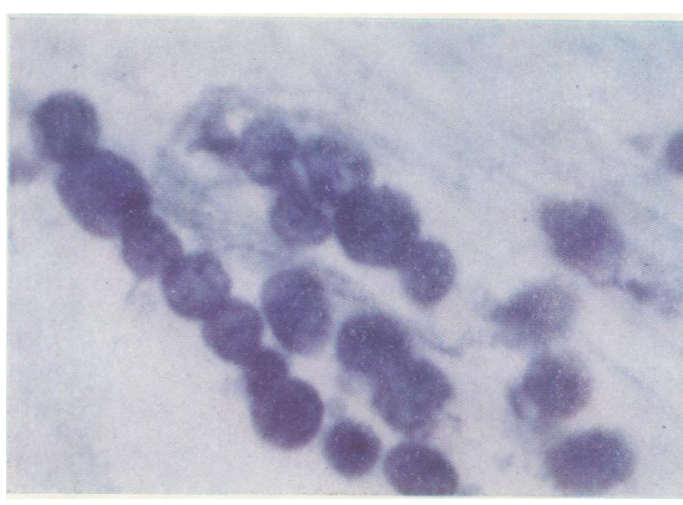

FIG. 2b. Oat-cell carcinoma in sputum

formed. In those patients in whom the cell type wa: known, nearly one-half had squamous cell carcinoma? and in this group over two-thirds were first dis covered by sputum examination.

Several sputum samples were often submitted frons one patient and the figures already quoted refer too the results obtained from examining all of them. If however, only the first sputum samples had beeno examined from each patient, the two observers. would have detected $37 \%$ of all the 229 patients withor cancer of the bronchus. Of the 70 with squamous cell carcinoma, $44 \%$ would have been discovered.

The value of sputum examination is clearly ${ }_{0}^{\omega}$ greatest in patients in whom bronchoscopy has not revealed the diagnosis. Ninety-eight of the 229 subjects with cancer had bronchoscopy performed. Of these, 38 had no bronchoscopic evidence of tumour but in $71 \%$ of these subjects the sputum revealed the diagnosis. An analysis of the firstत्ठ sputum sample from all these bronchoscopically $\frac{?}{\mathbb{D}}$ negative patients would have shown $42 \%$ positive. 
The two observers made their preparations as circumstances dictated, no system being used to determine who should have the first selection. No statistically significant difference was evident in recovery rates, i.e., it did not appear that an observer's success in finding tumour cells was dependent on his having the first choice of material.

An attempt was made to assess what advantage was gained by examining a second slide when the first one was found negative. In the case of observer 1 , the increased recovery rate was $14 \%$; in the case of observer $2,16 \%$. It is evident that the distribution of cancer cells in sputum is very erratic and that the success rate is therefore improved by examining more than one preparation.

The difference between the success of the two observers can be examined in two ways:

Sputum samples from patients with cancer of bronchus There were approximately 700 sputum samples and there was close agreement between the results of the two observers (approximately $29 \%$ positive).

Positive sputum samples from patients with cancer of bronchus. There were altogether 156 patients in this category, of whom observer 1 recorded $46 \%$ positive and observer $2,54 \%$. Both observers found a higher proportion positive among patients in whom the diagnosis was not histologically confirmed.

We tried to estimate the advantage of examining several sputum samples from each patient. One or both observers found cancer cells in the first sample in $37 \%$ of patients with carcinoma of the bronchus, $53 \%$ after two samples, $60 \%$ after three, $68 \%$ after four, and $71 \%$ after five samples. There is evidently a material gain with each additional examination until four samples have been scrutinized. Thereafter the additional gain is scarcely commensurate with the effort.

Observer 1, with several years' experience of this work, did not significantly alter his performance during the period of the trial. The second observer (with less experience) appeared to gain additional skill, or possibly confidence, after the passage of a few months, his recovery rate rising from $23 \%$ in the first half of the trial to $33 \%$ in the second half.

Results of sputum examination were compared from patients with peripheral cancers and those with central ones. One or both observers detected $61 \%$ (55 out of 90 subjects) known to have a central growth, $61 \%$ (22 out of 36 subjects) with a peripheral growth, and $59 \%$ (63 out of 107 subjects) in whom the position of the tumour was not known. In this context, the most interesting tumours are the peripheral, for they are beyond the reach of the bronchoscope. Of the 36 known to be peripheral, nearly two-thirds were diagnosed by exfoliated cancer cells in the sputum.
We were not very reliable in identifying the histological type of the exfoliated cells. In squamous cell carcinoma, the most frequent tumour present, our findings agreed with the histological results approximately three times out of four. In the other, less common, varieties of cancer the concurrence was of the order of $50 \%$.

The survey included 302 patients who were deemed clinically not to have cancer at the time of analysis. In 12 of these patients we reported the presence of cancer cells and three of them were subsequently shown to have a carcinoma of the bronchus. The other nine patients have not as yet shown any clinical evidence of neoplasm. Only one of the nine subjects was reported positive on more than one occasion. Our maximum inaccuracy therefore was $3 \%$ and it may finally prove to have been smaller. There is always the possibility that false positives may be due to exfoliation of cells from a tumour in the upper respiratory tract or mouth. Actual false positives may be due to inexperience in recognizing artefacts or cells derived from areas of squamous metaplasia in longstanding bronchiectatic or tuberculous cavities, or in patients who have formerly had a lobectomy or pneumonectomy. The bizarre appearances of degenerating bronchial epithelium have made us very uneasy about examining sputum for a period of 10 to 14 days after bronchoscopy. The presence of cilia is often of help in identifying bronchial epithelium derived from such traumatised mucosa.

CONCLUSion Had we known the clinical and other details at the time of examining the specimens, our results might have been somewhat more accurate, but this source of bias was deliberately avoided in an effort to clarify some aspects of the problem. The usefulness and reliability of this type of examination has long since been established but it involves a great deal of work by carefully trained observers. In view of this very serious drawback, clinicians should be urged to use this service to best advantage, by carefully selecting patients from whom sputum is submitted and seeing that proper, fresh, clean sputum samples are provided, free from extraneous debris. The procedure is most helpful in patients in whom there is a strong clinical presumption of cancer but in whom bronchoscopy is undesirable or has failed, possibly because there may be a tumour beyond the reach of the bronchoscope. In such patients it seems worth examining at least four or five sputum samples.

BLOOD

During the last few years I have been cooperating with Dr. J. R. Bignall, of the Institute of Diseases of 


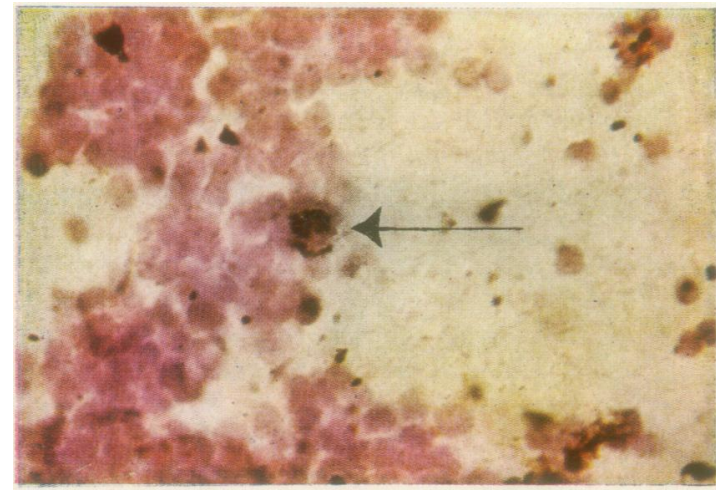

FIG. 3a. Clump of cancer cells from the pulmonary vein of a patient with poorly differentiated carcinoma of the bronchus showing autoradiograph of one of the cells.

the Chest, in examining methods for detecting exfoliated cancer cells in blood. We sought a reliable quantitative technique in the hope that it would be possible to test the effect of operative techniques and cytotoxic drugs on the numbers of cells exfoliated. Quantitative methods rely on filtration through a millipore membrane. Most of the techniques described rely mainly on lysis or on differential specific gravities, e.g., streptolysin or concentrated solutions of bovine albumin, or mixtures of silicones. In our hands the specific gravity methods were not really quantitative and reproducible, and the lytic technique was too costly. Finally we elaborated a new technique (Kuper, Bignall, and Luckcock, 1961).

Red blood cells are lysed by a brief exposure to a low concentration of saponin, but it is necessary to remove many of the white blood cells if a reasonably large volume of blood is to be filtered through a millipore membrane without crowding the membrane with normal cells. The removal from the blood of the polymorphonuclear cells achieves the necessary reduction in numbers, and we accomplished this by a modification of a method described by Cassen, Hitt, and Hays (1958) for causing these cells to phagocytose iron particles. The cells can then be removed by a magnet or by centrifugation. We chose the former, to ensure that any tumour cells were not entangled in the aggregates of engorged polymorphonuclear cells.

The choice of anticoagulant is important and satisfactory results are obtained only with heparin. We add a pinch of carbonyl iron (grade S. F.) to $10 \mathrm{ml}$. of heparinized blood and rotate it gently at $37^{\circ} \mathrm{C}$. for 30 minutes. The red cells are then lysed with $0.5 \%$ saponin and the fluid is swirled around the poles of a strong permanent magnet. Centrifugation is then necessary in order to remove the supernatant plasma proteins before the fixative is added (formalin and acetic acid in alcohol). The fluid is filtered through a millipore membrane which is

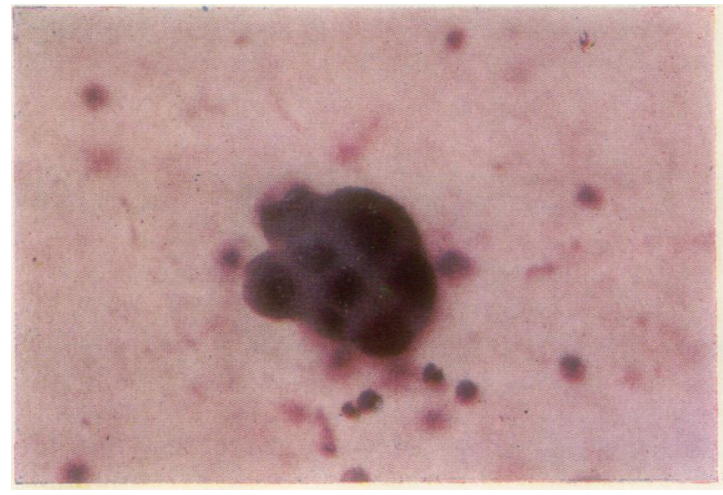

FIG. 3b. Clump of tumour cells from circulating hlood of $\omega$ patient with a neoplasm of the renal pelvis. (Autoradiographs. not made.)

then stained with haematoxylin and eosin, dehydrated, and mounted.

Recovery tests were done and the method was found to give reproducible results and to be quantitative. Five millilitres of normal blood processed in this way and filtered through a $47 \mathrm{~mm}$. membrane yields an acceptable level of cells in the background (lymphocytes). At one stage we hoped to be able to remove these cells but finally decided that they were not wholly undesirable, for they provide a scale for the assessment of size of other cells present.

A large number of blood samples were examined by this method. Some were from patients with neoplasms and others from patients thought to be free from malignant disease. Some of the samples were taken from a peripheral vein and some at operation from veins draining the site of a tumour (Fig. 3).

Alexander and Spriggs (1960) have drawn attention to the unexpected cell types that may be found when large volumes of blood are examined. It seems likely that megakaryocytes and immature blood cells have been mistaken for tumour cells by some of the early workers in this field. This fact may help to explain why our figures for recovery of tumour cells are lower than those in many of the published series. In fact, all preparations were assessed without knowledge of the source of the blood sample and the utmost caution was exercised before positive results were recorded.

RESULTS Two hundred and sixty-nine peripheral blood samples were taken from 91 patients with cancer of the bladder. Twenty per cent of these were considered to have cells in them probably derived from a tumour. No striking difference was found between the percentage recovered before, during, or 
after operation. Ninety-five peripheral vein samples were collected from 70 patients with carcinoma of the bronchus; only $4 \%$ of these were positive. In the case of pulmonary vein blood, 55 samples derived from 44 patients gave a $14 \%$ positive recovery. One hundred and eighty-nine peripheral blood samples were collected from 88 patients with cancer of the breast; $8 \%$ of these were positive whereas with regional vein blood 80 samples (from 21 patients) showed a $19 \%$ positive rate.

Engell (1959) has been studying this subject for many years and he introduced a note of scepticism concerning the significance of these cancer cells when he published some of the results of his five-to-nineyear survival rates. The apparent health of many of these patients so long after the recovery of tumour cells from their blood suggested the possibility that these cells might not be closely related to the occurrence of metastases. If, however, one compares the survival rates of his patients who had tumour cells in the blood with those who did not a striking difference is apparent. For example, if the group with carcinoma of the rectum is considered $29 \%$ of patients with tumour cells in the blood had survived, whereas $63 \%$ survived of those who did not have tumour cells. In his other groups the trend was similar though less striking.

Several animal experiments have been conducted in an effort to clarify certain aspects of the problem. Mr. Sellwood, in experiments with batches of mice, has shown the striking relationship between the number of tumour cells introduced into the circulation and the number of implantation tumours that result. Indeed, if 10,000 cells per animal were injected none of the mice developed tumours; with ten-fold increases, the number of animals affected rose steadily, and in similar experiments in rats both the number of animals with tumours and the number of tumours per animal showed a similar trend. These results will be published fully in due course.

At this juncture, Professor Smithers suggested that we might gain additional useful information if we were able to label recovered tumour cells with thymidine. This substance is necessary for the formation of D.N.A. which is synthesized in nuclei during the pre-mitotic phase. The uptake of thymidine by such cells is thus a clear indication of D.N.A. synthesis. Elaboration of a technique using millipore membranes confronted us with several difficulties, but a method has finally been worked out.

Tritiated thymidine is added in a concentration of $1.5 \mu \mathrm{c}$ per millilitre to the heparinized blood sample. After two and a half hours' incubation at $37^{\circ} \mathrm{C}$. a pinch of carbonyl iron is added and incubation continued for a further half hour. The blood sample is then treated as already described up to the stage when the material has been filtered through a millipore membrane. This is placed on a waxed microscope slide with a wheel-shaped magnet underneath the slide and a bevelled steel ring on its upper surface, so that the membrane is held in position by attraction between the magnet and the ring. In the dark room dilute photographic dipping emulsion is pipetted onto the surface of the membrane and the surplus allowed to run off. After drying in a stream of filtered air, the membrane is removed, placed in a light-proof container and stored in the refrigerator for a week. It is then developed and fixed as though it were a photographic negative, and stained with haematoxylin and eosin in the usual way. If any of the cells have taken up tritiated thymidine, this is evidenced by tiny black dots lying in the emulsion over these cells. They are, of course, grains of silver which have been exposed by radioactivity.

The technique outlined was developed using serous effusions containing tumour cells. This was considered necessary because of the inconstancy of tumour cells in blood samples and the very small numbers of cells which are found. The method was then adapted for use with blood samples, and Fig. 3a shows the result obtained with a blood sample from the pulmonary vein taken during pneumonectomy for carcinoma of the bronchus. At that time the incubation period with thymidine was one hour. Since then, we have ascertained that satisfactory results are obtainable after three hours' incubation of blood with thymidine. The $p \mathrm{H}$ and oxygen tension do not alter much, and we hope that this period of incubation will enable us to label a very much higher proportion of dividing cells than is provided by one hour's incubation. We have measured the percentage labelling by this technique, using the blood of healthy subjects, and only 2 or 3 cells per 1,000 recovered on the membrane yield autoradiographs. By contrast, blood samples from patients with Hodgkin's disease have shown figures of 10 to $15 \%$ for labelled cells (This percentage refers of course only to those cells recovered on the millipore membrane, namely, lymphocytes and primitive cells.)

\section{PLEURAL FLUID}

We used samples of pleural fluid extensively in trying to evolve a satisfactory technique for processing blood samples. A large proportion of the fluids obtained were from patients with cancer, many of whom had neoplastic lesions involving the pleura. During the scrutiny of millipore membranes used for filtering these fluids, it began to appear that frankly neoplastic effusions produced quite different autoradiographs from the effusions containing only mesothelial cells. The morphological differentiation between mesothelial and tumour cells has long been a matter of great difficulty and even now we must be somewhat uncertain in our estimation because of the 


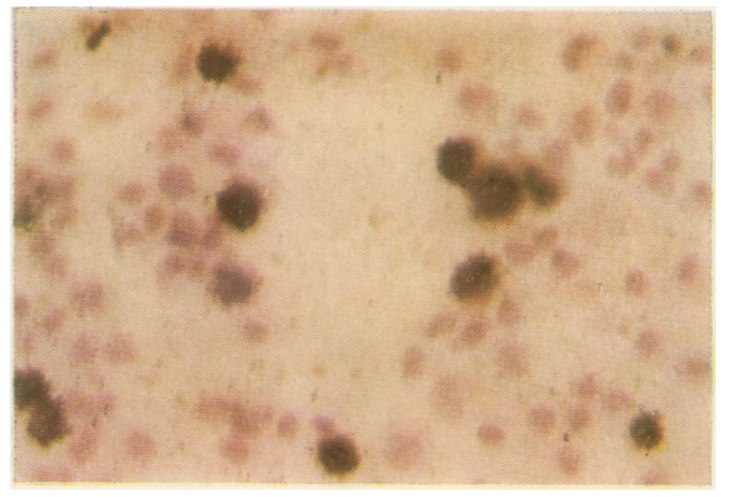

FIG. 4a. Millipore membrane preparation showing large numbers of tumour cells in pleural fluid with autoradiographs

inherent difficulty in deciding whether certain fluids do or do not contain neoplastic cells. The issue frequently remains in doubt and we have no certain way of proving our estimation right or wrong. We do, however, have a strong impression that the method to be described may be useful in assisting in this difficult decision.

Clumps of cells, frequently with some degree of differentiation, provide no great diagnostic difficulty. These morulae are identifiable on morphological grounds but we consider it necessary to prepare histological sections of cell deposits to ensure that they are not overlooked. We find it of great assistance when processing such a button of cells to do a final centrifugation in tiny plastic tubes. After hard centrifugation, the supernatant fluid is discarded and replaced with Bouin's fluid. If the cell button does not retract from the walls of the tube, it can be freed without disturbance by dissolving the tube in warm chloroform. It can then be taken through the usual histological section-cutting procedure.

A more difficult problem is the distinction between mesothelial and tumour cells occurring singly or in pairs. Mr. Sellwood suggested that we investigate the affinity of mesothelium for carbonyl iron. Mesothelial cells were found to be actively phagocytic but no known tumour cell showed this propensity.

A portion of the fluid was exposed to carbonyl iron in the incubator for half an hour and at the end of that period the iron-laden mesothelium was removed with a magnet. During this period, and for a further half hour thereafter, cells in the fluid were given an opportunity of absorbing tritiated thymidine. Those in the pre-mitotic phase of absorption utilize the thymidine to synthesize D.N.A. An additional sample was incubated with thymi-

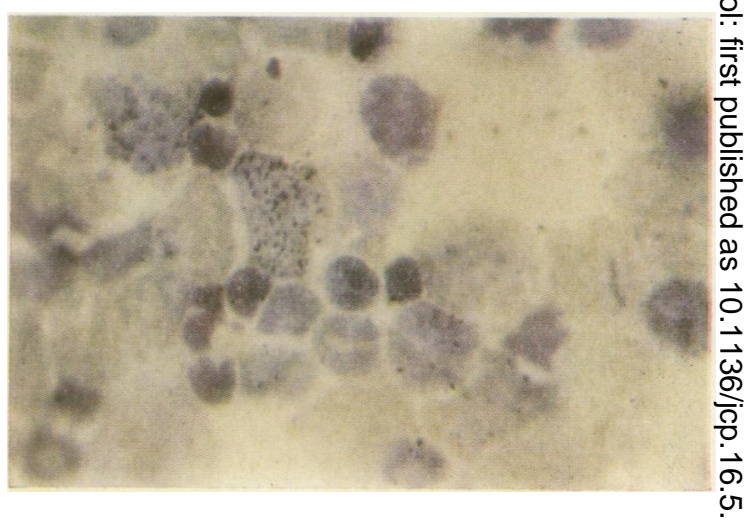

FIG. 4b. Smear preparation from centrifuged deposit of pleural fluid showing some tumour cells with autoradiographs

dine, but removal of the mesothelial cells was not attempted. After filtration through millipore membranes, autoradiographs were prepared. In addition, smears were made of the centifruged deposit after the cells had been washed several times in physiological saline to remove free $\overrightarrow{\mathscr{C}}$ thymidine. These smears were coated similarly with photographic emulsion. After three to seven days' storage in the dark in the refrigerator, the smears and membranes were developed and stained.

In preparations made from those fluids which we know to contain only mesothelium, the carbonyl iron technique effectively removed a large proportion of $\overrightarrow{\bar{\sigma}}$ the cells, and autoradiographs were only rarely $\exists$ observed. By contrast, cells from known malignant effusions frequently produced dramatic autoradiographs, several labelled cells sometimes being found in a single oil immersion field (Fig. 4). Though we cannot claim to have proved that this is a completely reliable technique for differentiating between the two types of cell, we have a strong impression that it may prove a valuable aid and further work is in hand in an effort to establish the facts.

I would like to express the thanks of Dr. Hinson and myself for the cooperation and assistance of the surgeons $\mathrm{N}$ of the Royal Marsden Hospital, Hammersmith Hospital, $N$ and Brompton Hospital, and to Mr. P. M. Payne, Director of the South Metropolitan Cancer Registry, for $\mathrm{\omega}$ statistical help.

\section{REFERENCES}

Alexander, R. F., and Spriggs, A. I. (1960). J. clin. Path., 13, 414. Cassen, B., Hitt, J., and Hays, E. F. (1958). J. Lab. clin. Med., 52, 778 . Engell, H. C. (1959). Ann. Surg., 149, 457.

Hinson, K. F. W., and Kuper, S. W. A. (1963). Thorax, in press Kuper, S. W. A., Bignall, J. R.. and Luckcock. E. D. (1961). Lancet 1, 852 . 